\title{
Improved Accuracy of Continuous Glucose Monitoring Systems in Pediatric Patients with Diabetes Mellitus: Results from Two Studies
}

\author{
Lori Laffel, MD, MPH
}

\begin{abstract}
Objective: This study was designed to evaluate accuracy, performance, and safety of the Dexcom (San Diego, CA) $\mathrm{G} 4{ }^{\circledR}$ Platinum continuous glucose monitoring (CGM) system (G4P) compared with the Dexcom G4 Platinum with Software 505 algorithm (SW505) when used as adjunctive management to blood glucose (BG) monitoring over a 7-day period in youth, 2-17 years of age, with diabetes.

Research Design and Methods: Youth wore either one or two sensors placed on the abdomen or upper buttocks for 7 days, calibrating the device twice daily with a uniform BG meter. Participants had one in-clinic session on Day 1, 4, or 7, during which fingerstick BG measurements (self-monitoring of blood glucose [SMBG]) were obtained every $30 \pm 5 \mathrm{~min}$ for comparison with CGM, and in youth 6-17 years of age, reference YSI glucose measurements were obtained from arterialized venous blood collected every $15 \pm 5$ min for comparison with CGM. The sensor was removed by the participant/family after 7 days.

Results: In comparison of 2,922 temporally paired points of CGM with the reference YSI measurement for G4P and 2,262 paired points for SW505, the mean absolute relative difference (MARD) was 17\% for G4P versus $10 \%$ for SW505 $(P<0.0001)$. In comparison of 16,318 temporally paired points of CGM with SMBG for G4P and 4,264 paired points for SW505, MARD was $15 \%$ for G4P versus $13 \%$ for SW505 $(P<0.0001)$. Similarly, error grid analyses indicated superior performance with SW505 compared with G4P in comparison of CGM with YSI and CGM with SMBG results, with greater percentages of SW505 results falling within error grid Zone A or the combined Zones A plus B. There were no serious adverse events or device-related serious adverse events for either the G4P or the SW505, and there was no sensor breakoff.

Conclusions: The updated algorithm offers substantial improvements in accuracy and performance in pediatric patients with diabetes. Use of CGM with improved performance has potential to increase glucose time in range and improve glycemic outcomes for youth.
\end{abstract}

\section{Introduction}

G LYCEMIC CONTROL REMAINS SUBOPTIMAL in pediatric patients with diabetes, with fewer than $25 \%$ of youth with type 1 diabetes (T1D) achieving glycemic targets of hemoglobin A1c (HbA1c) of $<7.5 \% .^{1-4}$ Use of advanced diabetes technologies, like continuous glucose monitoring (CGM) devices, has been associated with improved glycemic control $^{5-8}$ in some populations, like adults with T1D, but not universally, as pediatric samples have not experienced uniform benefits. ${ }^{5,9-14}$ Improved glycemic outcomes with CGM have been associated with consistent use of CGM. ${ }^{5,15,16}$ Thus, the lack of glycemic benefit of CGM among youth with
T1D has been attributed to inconsistent use. In particular, only $30 \%$ of $15-24$-year-old patients and $50 \%$ of 8-14-yearold patients in the JDRF CGM study wore CGM devices consistently for 6 or more days per week for the 6-month study duration compared with $86 \%$ of those 25 years of age and older. ${ }^{5}$ The latter group experienced a significant improvement in HbA1c compared with the control group using blood glucose (BG) monitoring alone. Notably, the devices used in these initial trials used early-generation technologies with accuracy, reliability, and usability issues.

The lack of consistent CGM use in the pediatric population has been attributed to several barriers associated with CGM. These barriers have been particularly evident in some of the 
first clinically available CGM devices, with barriers, for example, related to suboptimal accuracy and highly variable performance. ${ }^{17,18}$ These inaccuracies were attributable to many causes, including the time lag between interstitial and capillary glucose measurements and suboptimal algorithms that were used to derive the real-time glucose levels displayed on the CGM receiver. These challenges are especially evident in the pediatric population, who experience much greater glycemic variability and wider glycemic excursions than their adult counterparts with T1D. ${ }^{19,20}$ Indeed, it is not surprising that durability of CGM use has been modest in the pediatric population. The Type 1 Diabetes Exchange (T1DX) has documented limited CGM use, especially with the older CGM devices, as only $2-3 \%$ of pediatric patients $2-5,6-12$, and 13-17 years of age were using CGM in 2010-2012. ${ }^{2}$ A recent publication from the T1DX reports increased although still very limited uptake of CGM in pediatric patients, with only $13 \%$ of $2-5$ year olds, $8 \%$ of $6-12$ year olds, and $5 \%$ of 13-17 year olds using CGM. ${ }^{4}$

One of the main reasons for CGM discontinuation among the early adopting pediatric patients with T1D and their families related to sensor inaccuracies, leading to frustration when CGM and BG meter readings differed and when socalled "nuisance" CGM alerts and alarms were frequent. ${ }^{17,21}$ The temporal increase in CGM uptake documented in the T1DX coincides with improved accuracy of CGM devices, ${ }^{22}$ for example, related to the availability of the Dexcom G4 ${ }^{\circledR}$ Platinum compared with the earlier Dexcom STS ${ }^{\mathrm{TM}}$ or Seven ${ }^{\circledR}$ Plus systems. ${ }^{23}$ Notably, CGM users compared with nonusers had better glycemic control in the T1DX, independent of their form of insulin delivery, and this pattern was evident in the baseline registry data from 2010-2012 as well as in the most recently reported annual update covering the period 2013-2014. ${ }^{2,4}$ Therefore, improved CGM algorithm performance leading to better accuracy of CGM devices could likely lead to greater uptake and persistent use of CGM in the pediatric population, potentially yielding greater glucose time in range and improved glycemic control.

The current study aims to compare the CGM performance of two recently available CGM systems, the Dexcom G4 Platinum RT-CGM (G4P) and Dexcom G4 Platinum with Software 505 algorithm (SW505), in one of the largest pediatric accuracy reports to date, including more than 250 youth 2-17 years of age with T1D. The SW505 algorithm is the same algorithm as the one used in the recently released Dexcom G5 Mobile ${ }^{\mathrm{TM}}$ CGM system for pediatric patients, 2 years of age and older, as well as adult patients. It is important to study critically the accuracy and performance as well as safety for regulatory approval, but it also necessary to report to the clinical community substantive improvements in CGM performance in order to advance efforts at increasing CGM uptake aimed at optimizing control in the very vulnerable pediatric population in need of improved glycemic outcomes in order to preserve health and prevent both short- and longterm complications of diabetes.

\section{Research Design and Methods}

This report includes two studies of CGM accuracy in pediatric patients with diabetes, 2-17 years of age. Both studies were short-term 1-week open-label single-arm multicenter studies including youth treated with multiple daily injections or insulin pump therapy. In the first study of the G4P device involving six centers (Study 1), youth could have T1D or type 2 diabetes (only one of 176 patients studied had type 2 diabetes). In the second study of the SW505 algorithm involving five centers (Study 2), all youth had T1D. Exclusion criteria included hematocrits beyond the range recommended by the study glucose meters, pregnancy, hypoglycemic unawareness (other than that usually expected for toddlers with diabetes), need for treatment with acetaminophen, and any significant illness that would pose a risk to the patient or to the staff handling the blood specimen.

In Study 1, participants wore two CGM systems simultaneously for a 7-day sensor wear period (amounting to $168 \mathrm{~h}$ ), with one receiver providing real-time data and the other masked. In Study 2, participants wore a single unmasked sensor for the 7-day sensor wear period with CGM data displayed in real time. Sensors were inserted in the abdomen and/or upper buttocks by the patients or parents/guardians after self-training using a tutorial and/or one-on-one training by study staff.

Subjects in both studies were required to use a studyprovided BG meter (the LifeScan [Milpitas, CA] OneTouch ${ }^{\circledR}$ Verio ${ }^{\circledR}$ IQ in Study 1 and the Bayer [Whippany, NJ] Contour $^{\mathrm{TM}}$ Next USB BG meter in Study 2) and study-provided BG test strips for all BG measurements performed during sensor wear. In both studies, participants were asked to perform a minimum of seven fingersticks per day for home use for calibration (performed twice daily per labeling recommendations), comparative purposes, and all insulin dose selections as well as any other diabetes management decisions. All subjects avoided use of acetaminophen during the sensor wear period and for at least $24 \mathrm{~h}$ prior to sensor insertion. In both studies, participants were required not to inject insulin or wear an insulin pump insertion set within 3 inches of the sensor sites during wear. During home use, although the CGM data were displayed on the receiver screen, participants and families were to base all diabetes management decisions on results from the BG meter.

In both studies, subjects 6-17 years of age participated in one in-clinic session on either Day 1, 4, or 7 of sensor wear to allow for comparison of both the G4P and SW505 sensor glucose measurements with a reference glucose measurement (YSI BG analyzer; YSI, Yellow Springs, OH) obtained every $15 \pm 5$ min using arterialized venous blood and with glucose meter results using fingerstick capillary samples obtained every $30 \pm 5 \mathrm{~min}$. For youth $2-5$ years of age, only fingerstick capillary samples were obtained every $30 \pm 5 \mathrm{~min}$. The CGM systems were calibrated using BG meter results at the start of the clinic session. During the in-clinic sessions, all receivers were masked, avoiding any display of sensor glucose results.

The preschool-aged subjects (2-5 years of age) participated in a 4-h clinic session in both studies. School-aged subjects (6-12 years of age) participated in the clinic session for up to $6 \mathrm{~h}$. Teen subjects (13-17 years of age) participated in the clinic session for up to $6 \mathrm{~h}$ in Study 1 and for up to $12 \mathrm{~h}$ in Study 2. Those 6-17 years of age underwent intravenous catheterization of the dorsal hand, lower arm, or antecubital region for venous blood sampling for YSI plasma glucose determinations. The intravenous site was covered with a heating pad in order to arterialize the blood to more closely match the capillary BG concentrations. YSI sampling did not exceed $3 \mathrm{~mL} / \mathrm{kg}$ of each subject's body weight. In the teen 
sample in Study 2, glucose levels during the in-clinic session were manipulated under close supervision according to protocol guidelines in efforts to achieve glucose levels across the range of sensor performance $(40-400 \mathrm{mg} / \mathrm{dL})$ by either adjusting the timing of meals (delaying meals after insulin administration to induce controlled hypoglycemia) or the timing of insulin administration (delaying insulin dosing with food intake to induce hyperglycemia). For youth of all ages in Study 1 and for those 2-12 years of age in Study 2, the participants checked their BG levels, took insulin, and ate as per their usual practice. At the end of the clinic session, study staff unmasked the receivers, and all receivers and glucose meters were downloaded using a sponsor-provided clinical laptop.

For both studies, on Day 7, participants returned to the clinic, removed the sensors themselves (or the sensors were removed by parents/guardians, as applicable), and returned the CGM systems and BG meters to the study staff. Study staff assessed adverse events related to study procedures, device use, and skin irritation. Staff carefully inspected the skin at the sites of sensor insertions and used the Draize scale to grade skin irritation. ${ }^{24}$ Sensors were inspected by the study staff and the sponsor to assess any sensor breakoff during use.

Both study protocols were approved by the site's Institutional Review Board or a central Institutional Review Board according to local policy, and all participants and parents/ guardians provided signed assent (as appropriate for the youth's age) and informed consent, respectively, prior to performance of any study procedures. Both studies were reviewed and approved by the Food and Drug Administration prior to performance through the Investigational Device Exemption process.

\section{Statistical methods and analyses}

All data are presented as mean \pm SD values or percentages unless otherwise indicated. Parametric (unpaired $t$ tests) and nonparametric (Wilcoxon tests) were used as indicated by the sample distribution of the means. For comparisons of categorical variables, $\chi^{2}$ tests were used.

The real-time CGM values were compared with the temporally matched glucose values from the reference YSI and BG meter values. The mean absolute relative difference (MARD), as well as median absolute relative difference (ARD), in percentages and the proportion of the CGM system values that were within $( \pm) 20 \%$ of the relative difference from the reference value at glucose levels $>80 \mathrm{mg} / \mathrm{dL}$ and within ( \pm ) $20 \mathrm{mg} / \mathrm{dL}$ of absolute difference at glucose levels $\leq 80 \mathrm{mg} / \mathrm{dL}$ (hereafter referred to as \%20/20) were used to evaluate the overall accuracy performance of the CGM devices. Similarly, performances of the G4P and SW505 systems were assessed within glucose ranges of 40-60 mg/dL, $61-80 \mathrm{mg} / \mathrm{dL}, 81-180 \mathrm{mg} / \mathrm{dL}$, and $>180 \mathrm{mg} / \mathrm{dL}$ as well as within each day of sensor use, from Day 1 through Day 7. The mean absolute difference (MAD), as well as median absolute difference, was also used to assess performance in the hypoglycemic range, $40-80 \mathrm{mg} / \mathrm{dL}$.

Modified Bland-Altman plots ${ }^{25}$ were used to depict the data distribution and bias between the CGM and the reference glucose determinations. Error grid analyses, including the Clarke Error Grid ${ }^{26}$ and Parkes Error Grid, ${ }^{27}$ were used to quantify the clinical accuracy of the CGM devices. CGM diagnostic features were evaluated in the hypoglycemic range $\leq 80 \mathrm{mg} / \mathrm{dL}$ and in the hyperglycemic range $\geq 240 \mathrm{mg} /$ $\mathrm{dL}$ by assessing concordance of CGM values within $15 \mathrm{~min}$ of the reference YSI results at these levels. Rate of both low and high false CGM alerts was also assessed based on CGM readings in the hypoglycemic or hyperglycemic ranges, respectively, when the matched YSI values within 15 min were not out of range. All analyses were performed using SAS software version 9.3 (SAS Institute, Inc., Cary, NC). $P$ values of $<0.05$ were considered statistically significant.

\section{Results}

\section{Participant characteristics and sensor usage}

Study 1 consisted of 176 subjects, with 29 in the 2-5-year age group, 69 in the 6-12-year age group, and 78 in the 1317-year age group, enrolled from six clinical centers in the United States. Study 2 included 79 subjects, with 16 in the 25-year age group, 17 in the 6-12-year age group, and 46 in the 13-17-year age group, enrolled from five clinical centers in the United States. Almost all patients had T1D, with an average duration about 5 years; the majority received insulin pump therapy. Mean HbA1c values were $8.2 \pm 1.3 \%$ and $8.5 \pm 1.5 \%$ in Studies 1 and 2, respectively. In Study 1, 40\% of participants had previous exposure to CGM, whereas only $13 \%$ used CGM devices on a routine basis; in Study 2, 57\% had previous exposure to CGM, whereas only $19 \%$ used it on a routine basis. Characteristics of the youth participants in Studies 1 and 2 are displayed in Table 1.

Table 1. Patient Characteristics in Studies of the Dexcom G4 Platinum Continuous Glucose

Monitoring System and the G4 Platinum WITH The Software 505 Algorithm

\begin{tabular}{lcc}
\hline & \multicolumn{2}{c}{ Study sample } \\
\cline { 2 - 3 } & $G 4 P$ & $\begin{array}{c}\text { SW505 } \\
(\mathrm{n}=79)\end{array}$ \\
\hline Age (years) & $11.4 \pm 4.2$ & $12.2 \pm 4.6$ \\
Diabetes duration (years) & $4.8 \pm 3.7$ & $5.6 \pm 4.2$ \\
z-BMI & & \\
Mean \pm SD & $0.5 \pm 1.0$ & $0.7 \pm 0.7$ \\
Range & -4.7 to 2.6 & -1.4 to 2.2 \\
SMBG (times/day) & $6.7 \pm 2.9$ & $6.7 \pm 2.3$ \\
HbA1c (\%) & $8.2 \pm 1.3$ & $8.5 \pm 1.5$ \\
Sex (\% male) & $57 \%$ & $52 \%$ \\
Race (\% white) & $94 \%$ & $96 \%$ \\
Type 1 diabetes (\%) & $99 \%$ & $100 \%$ \\
Pump use (\%) & $72 \%$ & $60 \%$ \\
Previous RT-CGM use (\%) & $40 \%$ & $57 \%$ \\
Frequent (50\% of time) & $13 \%$ & $19 \%$ \\
$\quad$ RT-CGM use (\%) & & \\
Age group (years) [n (\%)] & & $16(20 \%)$ \\
2-5 & $29(16 \%)$ & $17(22 \%)$ \\
6-12 & $69(39 \%)$ & $46(58 \%)$ \\
13-17 & $78(44 \%)$ & \\
\hline
\end{tabular}

${ }^{\text {a } O n e ~ s u b j e c t ~ w i t h ~ t y p e ~} 2$ diabetes, a 17-year-old female with type 2 diabetes duration of 3 years, multiple daily insulin injections therapy, and $12.1 \%$ hemoglobin A1c (HbAlc).

${ }^{\mathrm{b}}$ One subject with type 1 diabetes only used rapid-acting insulin correction scale for high glucose values but no long-acting insulin.

BMI, body mass index; G4P, G4 Platinum; RT-CGM, real-time continuous glucose monitoring; SMBG, self-monitoring of blood glucose; SW505, Software 505 algorithm. 
The expected number of sensors for use in both studies was $431(176 \times 2$ sensors/subject in Study 1 and $79 \times 1$ sensor/ subject in Study 2). Nearly all participants (244 out of 255, $96 \%$ ) succeeded in inserting and wearing the originally placed sensors; only 11 subjects required sensor replacements. In total, 443 sensors were issued, indicating that 12 sensors were replaced among these 11 study subjects. Data from only six sensors out of $431(1 \%)$ were excluded because of multiple reasons (e.g., deployment issues, inadvertent switch of transmitters between sib-pairs). Thus data were available from a total of 425 sensors in both studies. Of these, 367 sensors $(86 \%)$ lasted through Day 7; 412 sensors $(97 \%)$ lasted more than 4 days.

\section{CGM performance}

For assessment of CGM accuracy against YSI glucose measurements during the in-clinic sessions, there were 2,922 paired results (CGM and YSI temporally matched) in Study 1 and 2,262 paired results in Study 2 (Table 2). The overall MARD was $17 \%$ in Study 1 of the G4P, with a significant improvement in the MARD to $10 \%$ in Study 2 with the SW505 $(P<0.0001)$. Median ARD was $14 \%$ in Study 1 and $8 \%$ in Study 2. For assessment of CGM accuracy against selfmonitoring of $\mathrm{BG}(\mathrm{SMBG})$ meter results during the 1 week of sensor wear, there were 16,318 paired results (CGM and meter temporally matched) in Study 1 and 4,262 paired results in Study 2. The overall MARD was $15 \%$ in Study 1 and $13 \%$ in Study $2(P<.00001)$. The median ARD was $11 \%$ in Study 1 and $10 \%$ in Study 2. For both the G4P and SW505,
CGM accuracy improved after Day 1 of sensor use (Fig. 1). For CGM accuracy against the YSI, MARD improved from $21 \%$ on Day 1 to $16 \%$ on Day 4 and then to $15 \%$ on Day 7 for the G4P; MARD improved from $13 \%$ on Day 1 to $8 \%$ on Day 4 and then to $10 \%$ on Day 7 for the SW505. For CGM accuracy against SMBG, MARD improved from $19 \%$ on Day 1 to $12 \%$ on Day 7 for the G4P; MARD improved from $15 \%$ on Day 1 to $11 \%$ on Day 7 for the SW505.

Similarly, the Clarke Error Grid results indicated superior clinical accuracy with the SW505 algorithm compared with the G4P in the comparison of CGM versus YSI glucose values (Fig. 2a and b). In Study 1, 68\% of values fell in Zone A, and $98 \%$ fell in Zones A and B for the G4P; in Study 2, $90 \%$ of values fell in Zone A, and $99 \%$ fell in Zones A and B for the SW505 algorithm. In Study 1, for the comparison of CGM with SMBG values, $75 \%$ of values fell in Zone A, and 98\% fell in Zones A and B for the G4P (Fig. 2c); in Study 2, $86 \%$ fell in Zone A, and 98\% fell in Zones A and B for the SW505 (Fig. 2d). Assessment of CGM accuracy using the Parkes Error Grid (Fig. 3) yielded a similarly improved performance with the SW505 compared with the G4P, with greater percentages of CGM falling within the clinically accurate Zone A and the combined Zone A plus the benign error Zone B for CGM versus YSI (Fig. 3a and b) and CGM versus SMBG (Fig. 3c and d). Notably, $100 \%$ of the CGM values fell within Zones A and B for CGM versus YSI and for CGM versus SMBG with the SW505.

The \%20/20 was $68 \%$ for the G4P and $91 \%$ for the SW505 algorithm in the comparison of CGM with YSI; the \%20/20 was $76 \%$ for the G4P and $84 \%$ for the SW505 algorithm in

Table 2. Continuous Glucose Monitoring (CGM) Performance Accuracy During Clinic (CGM vs. Reference YSI) and Home (CGM vs. Self-Monitoring of Blood Glucose) Use

\begin{tabular}{|c|c|c|c|c|}
\hline & \multicolumn{2}{|c|}{$C G M$ versus $Y S I$} & \multicolumn{2}{|c|}{$C G M$ versus $S M B G$} \\
\hline & $G 4 P$ & SW505 & $G 4 P$ & SW505 \\
\hline Number of matched pairs & 2,922 & 2,262 & 16,318 & 4,264 \\
\hline Mean/median ARD (\%) & $17 / 14$ & $10 / 8$ & $15 / 11$ & $13 / 10$ \\
\hline CEG Zone A $(\%) / \mathrm{A}+\mathrm{B}(\%)$ & $68 / 98$ & 90/99 & $75 / 98$ & $83 / 98$ \\
\hline PEG Zone A $(\%) / \mathrm{A}+\mathrm{B}(\%)$ & $79 / 99$ & $93 / 100$ & $80 / 99$ & $86 / 100$ \\
\hline$\% 20 / 20 / \% 30 / 30(\%)$ & $68 / 85$ & $91 / 96$ & $76 / 89$ & $84 / 94$ \\
\hline \multicolumn{5}{|l|}{ Within CGM ranges } \\
\hline \multicolumn{5}{|l|}{$40 \leq \mathrm{CGM} \leq 60 \mathrm{mg} / \mathrm{dL}^{\mathrm{a}}$} \\
\hline Number of matched pairs & 19 & 86 & 487 & 240 \\
\hline Mean/median AD (mg/dL) & $19 / 9$ & $16 / 13$ & $24 / 18$ & $17 / 14$ \\
\hline \multicolumn{5}{|l|}{$60<\mathrm{CGM} \leq 80 \mathrm{mg} / \mathrm{dL}^{\mathrm{a}}$} \\
\hline Number of matched pairs & 76 & 142 & 1,340 & 399 \\
\hline Mean/median AD (mg/dL) & $13 / 11$ & $12 / 8$ & $17 / 11$ & $14 / 10$ \\
\hline \multicolumn{5}{|l|}{$80<\mathrm{CGM} \leq 180 \mathrm{mg} / \mathrm{dL}$} \\
\hline Number of matched pairs & 1,155 & 805 & 7,084 & 1,650 \\
\hline Mean/median ARD (\%) & $17 / 13$ & $11 / 8$ & $15 / 11$ & $12 / 9$ \\
\hline \multicolumn{5}{|l|}{$\mathrm{CGM}>180 \mathrm{mg} / \mathrm{dL}$} \\
\hline Number of matched pairs & 1,672 & 1,229 & 7,407 & 1,975 \\
\hline Mean/median ARD (\%) & $18 / 14$ & $9 / 7$ & $14 / 10$ & $11 / 8$ \\
\hline \multicolumn{5}{|l|}{$\mathrm{CGM}>250 \mathrm{mg} / \mathrm{dL}$} \\
\hline Number of matched pairs & 724 & 608 & 3,604 & 964 \\
\hline Mean/median ARD (\%) & $18 / 15$ & $10 / 7$ & $14 / 10$ & $11 / 8$ \\
\hline
\end{tabular}

${ }^{\mathrm{a}}$ Absolute differences $(\mathrm{AD}$; in $\mathrm{mg} / \mathrm{dL})$ included in these two ranges.

$\% 20 / 20 / \% 30 / 30$, values within $( \pm) 20 \%$ of the relative difference from the reference value at glucose levels $>80 \mathrm{mg} / \mathrm{dL}$ and within $( \pm) 20 \mathrm{mg} / \mathrm{dL}$ of absolute difference at glucose levels $\leq 80 \mathrm{mg} / \mathrm{dL} / \mathrm{values}$ within $( \pm) 30 \%$ of the relative difference from the reference value at glucose levels $>80 \mathrm{mg} / \mathrm{dL}$ and within $( \pm) 30 \mathrm{mg} / \mathrm{dL}$ of absolute difference at glucose levels $\leq 80 \mathrm{mg} / \mathrm{dL} ;$ ARD, absolute relative difference; CEG, Clarke Error Grid; G4P, G4 Platinum; PEG, Parkes Error Grid; SW505, Software 505 algorithm. 
G4P घGP with SW505

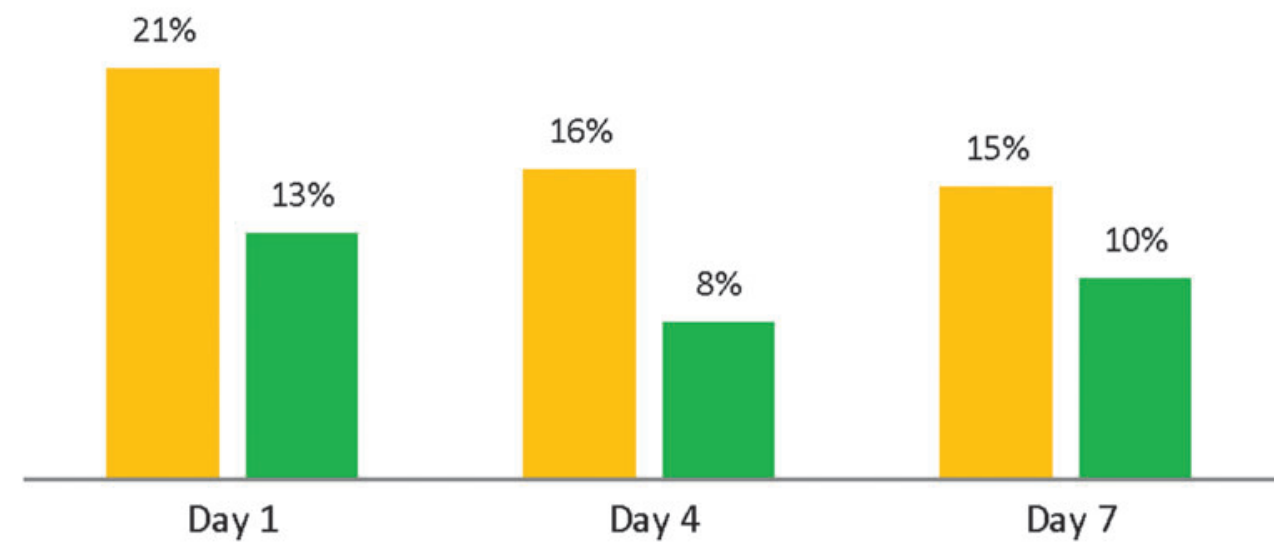

b MARD(\%) of CGM-SMBG Across Sensor Use Days

G4P G4P with SW505

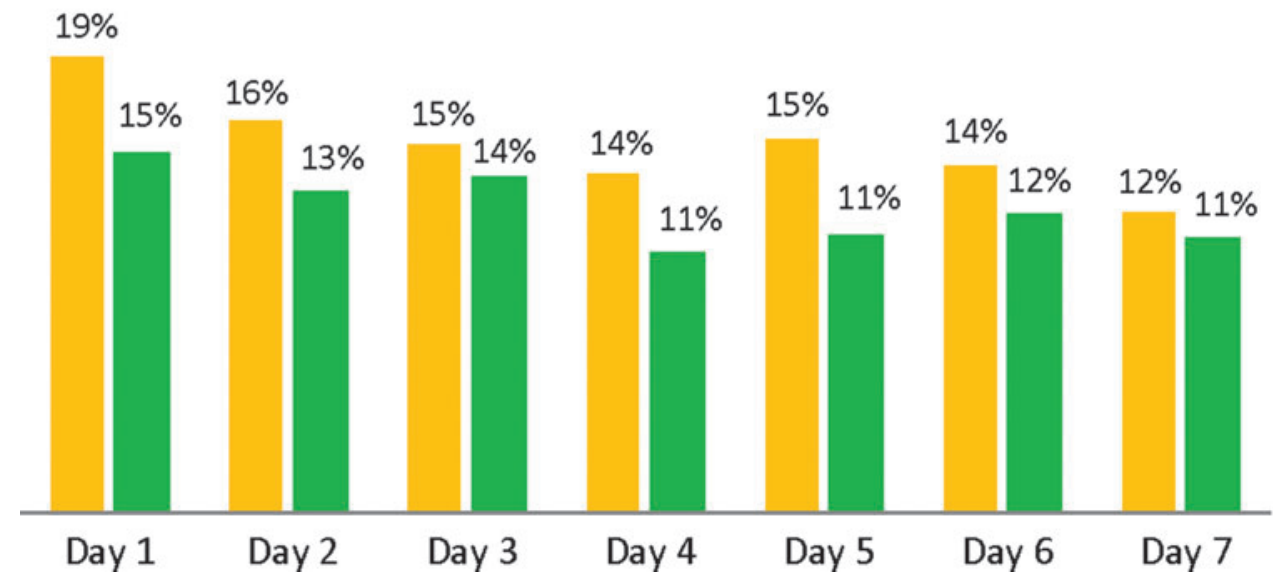

FIG. 1. Mean absolute relative difference (MARD) (\%) comparison of continuous glucose monitoring against (a) reference YSI across in-clinic study Days 1, 4, and 7 and (b) self-monitoring of blood glucose across home use Days 1-7. For continuous glucose monitoring accuracy against the YSI, MARD improved from 21\% on Day 1 to $16 \%$ on Day 4 and then to $15 \%$ on Day 7 for the G4 Platinum (G4P); MARD improved from 13\% on Day 1 to $8 \%$ on Day 4 and then to $10 \%$ on Day 7 for the Software 505 algorithm (SW505). For continuous glucose monitoring accuracy against self-monitoring of blood glucose, MARD improved from $19 \%$ on Day 1 to $12 \%$ on Day 7 for the G4P; MARD improved from $15 \%$ on Day 1 to $11 \%$ on Day 7 for the SW505.

the comparison of CGM with SMBG values. The $( \pm) 30 \%$ / $( \pm) 30 \mathrm{mg} / \mathrm{dL}$ values displayed the same pattern of improved performance accuracy for the SW505.

CGM performance was also assessed across various CGM glucose ranges where the SW505 performance was also superior to the G4P. Although the MARD was highest in the hypoglycemic range for glucose values $40-80 \mathrm{mg} / \mathrm{dL}$, inclusive, the MAD offers an alternative assessment of accuracy in this range. MARD values were $18 \%$ for G4P and $17 \%$ for the SW505 (comparison of CGM with YSI) and 21\% for the G4P and 19\% for the SW505 algorithm (comparison of CGM with SMBG). For the glucose values 40-60 mg/dL, the MAD values were $19 \mathrm{mg} / \mathrm{dL}$ and $16 \mathrm{mg} / \mathrm{dL}$ for the G4P and
SW505, respectively, in the comparison of CGM with YSI; the MAD values were $24 \mathrm{mg} / \mathrm{dL}$ and $17 \mathrm{mg} / \mathrm{dL}$ for the G4P and SW505, respectively, in the comparison of CGM with SMBG. For the glucose values $61-80 \mathrm{mg} / \mathrm{dL}$, the MAD values were $13 \mathrm{mg} / \mathrm{dL}$ and $12 \mathrm{mg} / \mathrm{dL}$ for the G4P and SW505, respectively, in the comparison of CGM with YSI; the MAD values were $17 \mathrm{mg} / \mathrm{dL}$ and $14 \mathrm{mg} / \mathrm{dL}$ for the G4P and SW505, respectively, in the comparison of CGM with SMBG. Across other glucose ranges, the SW505 performed substantially better than the G4P with MARDs of 9-12\% for the SW505 compared with 14-18\% for the G4P (Table 2).

Bland-Altman density plots depict the bias of CGM to YSI and of CGM to SMBG in $\mathrm{mg} / \mathrm{dL}$ for the G4P and the SW505 

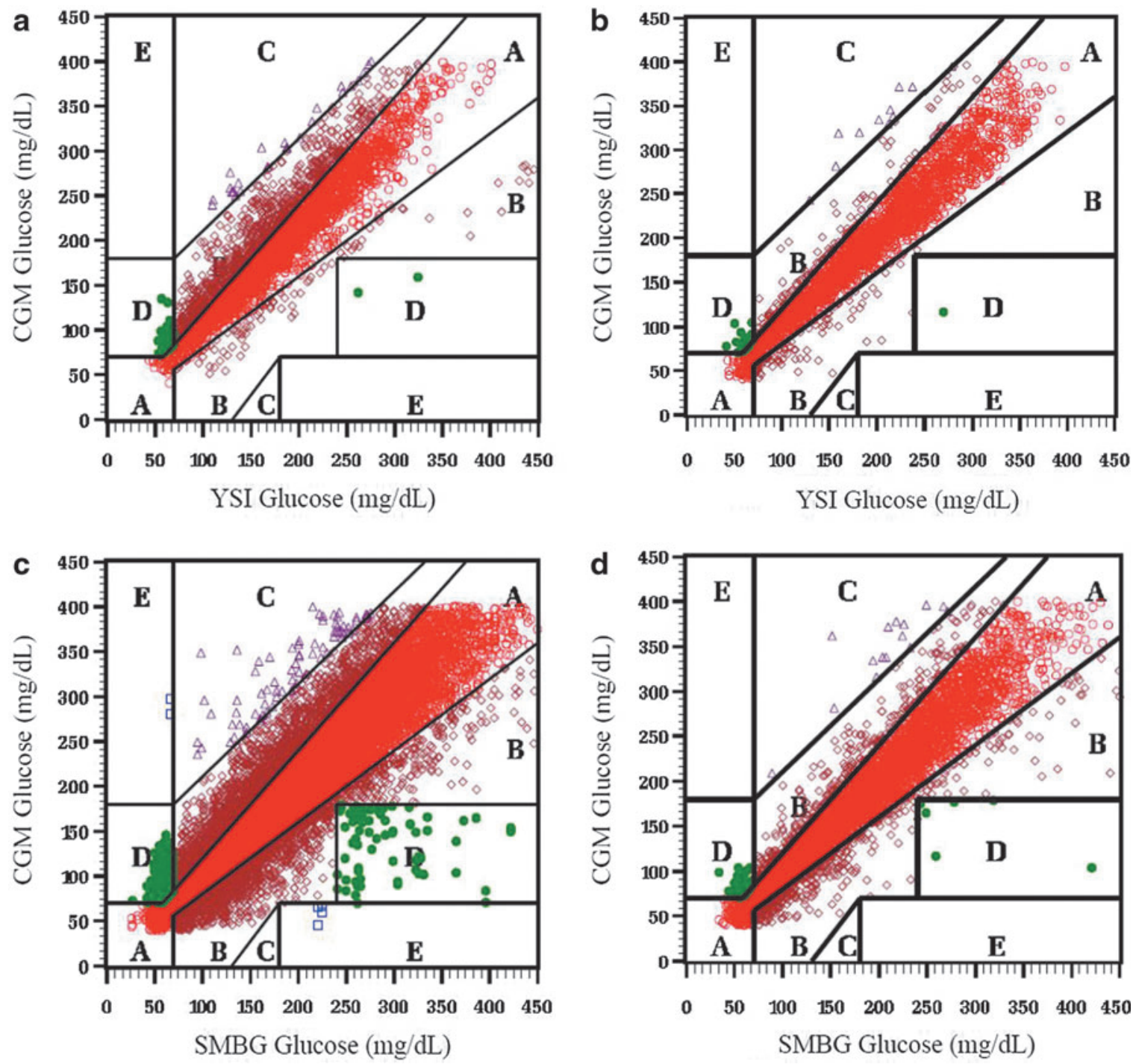

FIG. 2. Clarke Error Grids for continuous glucose monitoring (CGM) versus reference YSI (a and b) and self-monitoring of blood glucose (SMBG) (c and d) for the G4 Platinum (a and c) and G4P Platinum with Software 505 algorithm (b and d). The Clarke error grid results indicate superior performance with the Software 505 algorithm compared with the G4 Platinum in the comparison of CGM versus reference YSI glucose values and CGM versus SMBG. For the G4 Platinum, 68\% of values fell in Zone A, and 98\% fell in Zones A and B; for the Software 505 algorithm, 90\% of values fell in Zone A, and 99\% fell in Zones A and B. For the comparison of CGM with SMBG values, $75 \%$ of values fell in Zone A, and $98 \%$ fell in Zones A and B for the G4 Platinum; 86\% fell in Zone A, and 98\% fell in Zones A and B for the Software 505 algorithm.

(shown in Fig. 4). The bias was centered around 0 with higher density for the SW505 compared with the G4P for both CGM versus YSI and CGM for SMBG. The majority of the bias for the SW505 fell within the modified International Organization for Standardization area of \%20/20.

\section{CGM detection of hypoglycemia and hyperglycemia}

The SW505 performed superiorly to the G4P with respect to detection of hypo- and hyperglycemia. With a low glucose alert of $80 \mathrm{mg} / \mathrm{dL}, \mathrm{CGM}$ detected true hypoglycemia according to YSI measurements $\leq 80 \mathrm{mg} / \mathrm{dL} 55 \%$ of the time within 15 min with the G4P and $91 \%$ of the time within 15 min with the SW505. In this hypoglycemic range, there was a false alert rate of $34 \%$ with the G4P and $14 \%$ with the
SW505. With a high glucose alert of $240 \mathrm{mg} / \mathrm{dL}$, CGM detected true hyperglycemia according to YSI measurements $\geq 240 \mathrm{mg} / \mathrm{dL} 96 \%$ of the time within $15 \mathrm{~min}$ with the G4P and $94 \%$ of the time within $15 \mathrm{~min}$ with the SW505. In this hyperglycemic range, there was a false alert rate of $33 \%$ with the G4P and $12 \%$ with the SW505.

\section{Safety issues}

There were no serious adverse events or device-related serious adverse events for either the G4P or the SW505 among the pediatric patients in either study. There was no sensor break-off or infection at the site of sensor insertion. There was mild skin irritation in some patients in the adhesive area, occurring at a low rate. 

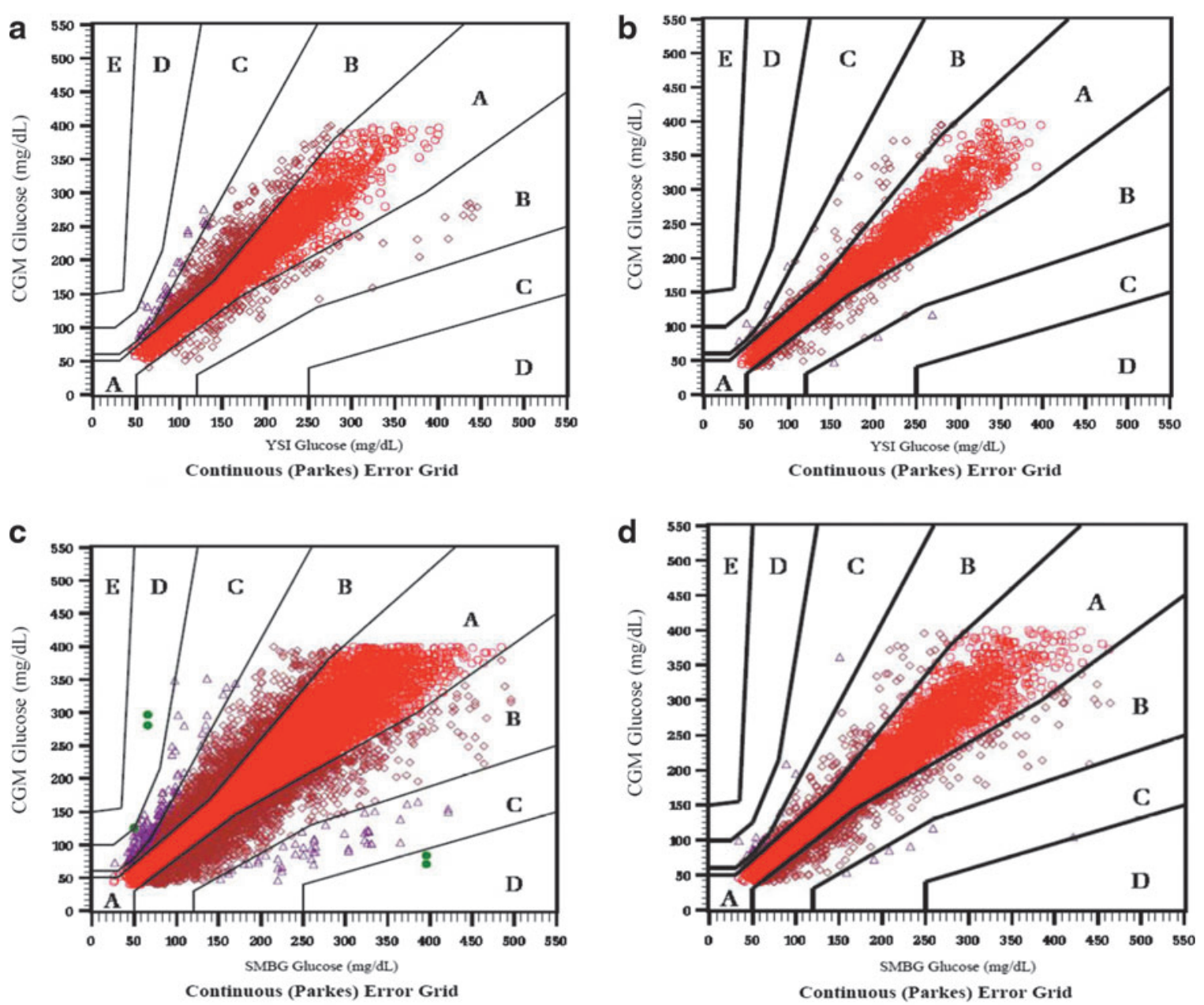

FIG. 3. Parkes Error Grids for continuous glucose monitoring (CGM) versus reference YSI (a and b) and self-monitoring of blood glucose (SMBG) (c and d) for the G4 Platinum (a and c) and G4P Platinum with Software 505 algorithm (b and d). Assessment of CGM accuracy using the Parkes Error Grid yielded improved performance with the Software 505 algorithm compared with the G4 Platinum, with greater percentages of CGM values falling within the clinically accurate Zone A and the combined Zone A plus the benign error Zone B for CGM versus reference YSI and CGM versus SMBG. Notably, 100\% of the CGM values fell with Zones A and B for CGM versus YSI and for CGM versus SMBG with the Software 505 algorithm.

\section{Conclusions}

This report describes the performance of the G4P CGM system as well as the improved performance of the G4P with the SW505 algorithm in pediatric patients with diabetes. The initial study (Study 1), evaluating the safety and efficacy of the G4P, provided data for the Food and Drug Administration approval of the device for adjunctive use in pediatric patients, 2 years of age and older, in February 2014. The second study (Study 2) evaluated the safety and efficacy of the G4P with the updated SW505 algorithm for use in pediatric patients, using the same algorithm as that used in the adult G4P device and the currently approved Dexcom G5 Mobile CGM system. Indeed, Study 2 provided the safety and efficacy data for the SW505 algorithm for Food and Drug Administration approval for use in pediatric patients 2 years of age and older. The G5 Mobile, with the same advanced algorithm, also received the European CE mark as of September 2015 for use in pediatric patients as young as 2 years old, based on the overall improved performance. In pediatric patients, the SW505 has an MARD of $10 \%$ compared with reference YSI values, closely matching the favorable MARD of $9 \%$ in adults with diabetes. ${ }^{28}$ Similarly, clinical accuracy based on error grid analyses yielded $98-100 \%$ of values falling within Zones A and B in the pediatric patients compared with $99.5 \%$ for adults, and $\% 20 / 20$ was $91 \%$ for the younger patients and 93\% for the adults for the SW505 in the comparisons of CGM with reference YSI glucose levels. ${ }^{28}$ Notably, the SW505 algorithm's performance has yielded opportunities for automated insulin delivery in many research settings. ${ }^{29,30}$

Although precision was not evaluated in Study 2 using the 505 algorithm, in Study 1 of the G4P, the overall paired absolute relative difference was $10 \%$, and the overall coefficient of variation was $7 \%$ (data not shown). In addition, precision according to sensor site (abdomen and buttocks) was also satisfactory, varying from $6.3 \%$ (both sensors on abdomen) to $7.5 \%$ (one sensor on abdomen and one on buttocks), with the coefficient of variation midway at $7 \%$ for two 
a

Bias Plot of Gen4 vs YSI Glucose Measurements

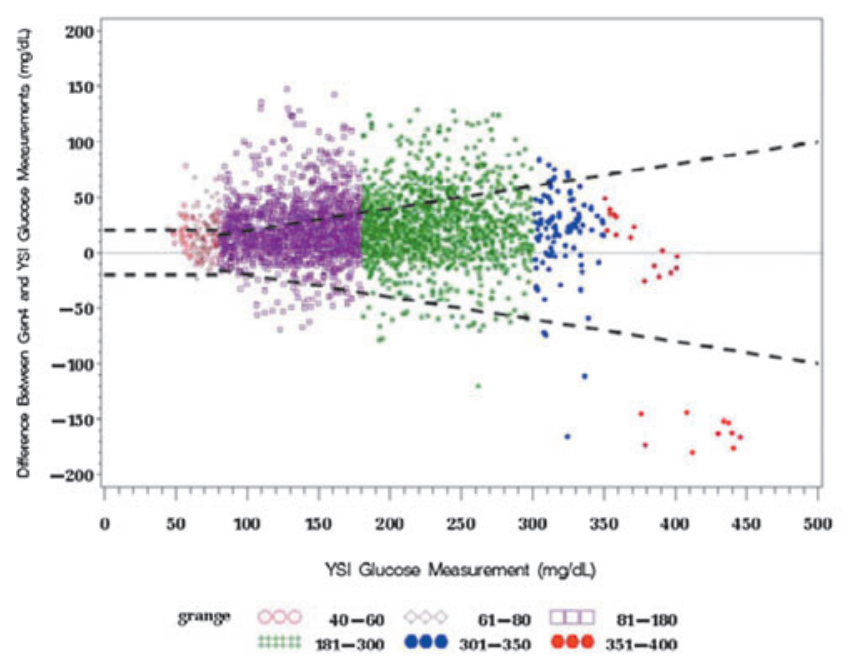

C

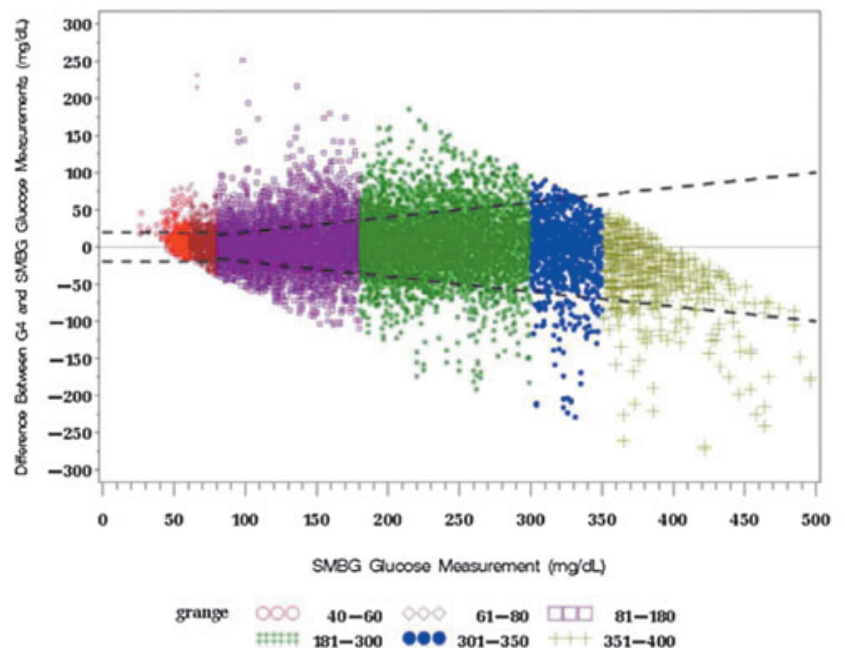

b

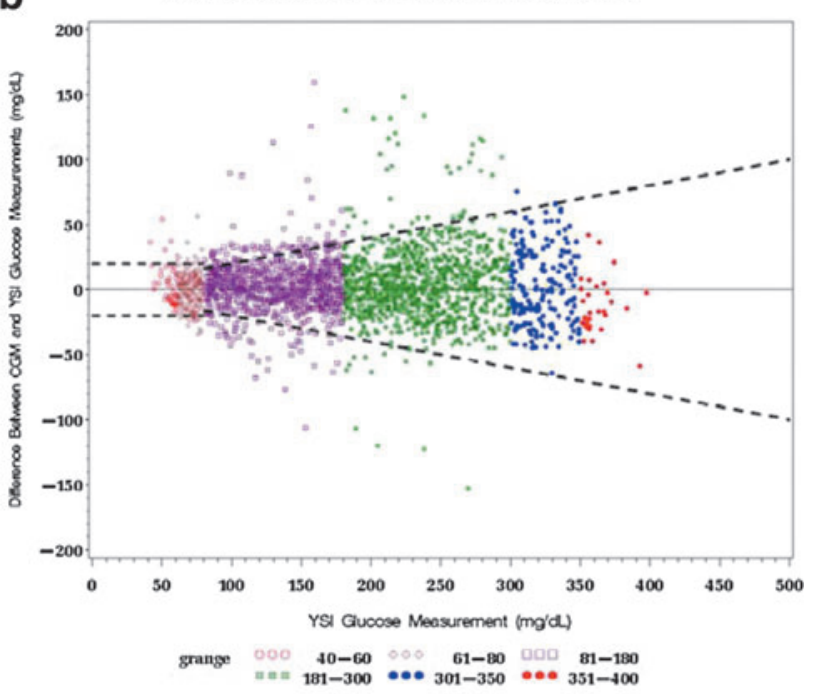

d

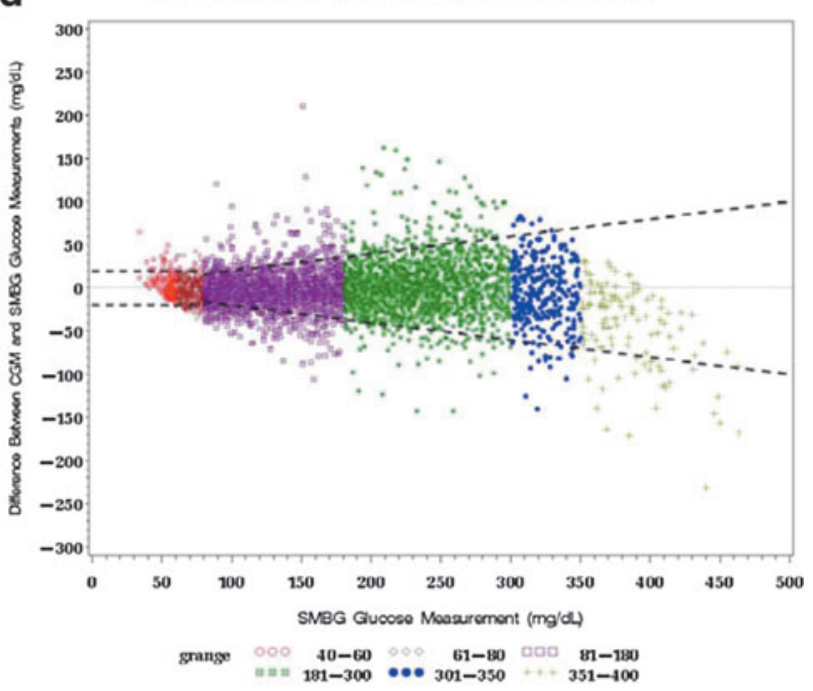

FIG. 4. Bland-Altman density plots of continuous glucose monitoring (CGM) versus reference YSI glucose (a and b) and self-monitoring of blood glucose (SMBG) (c and d) measurements for the G4 Platinum (a and c) and the G4 Platinum with Software 505 algorithm (b and d). In Bland-Altman density plots, depicting the bias of CGM to YSI and of CGM to SMBG (in $\mathrm{mg} / \mathrm{dL}$ ) for the G4 Platinum and the Software 505 algorithm, the bias was centered around 0 , with higher density for the Software 505 algorithm compared with the G4 Platinum for both CGM versus YSI and CGM for SMBG. The majority of the bias for the Software 505 algorithm fell within the modified International Organization for Standardization area of \%20/20.

sensors on the buttocks. Additionally, sensor performance for the G4P and the SW505 was fairly consistent across the pediatric age range, including the 2-5 year olds, the 6-12 year olds, and the 13-17 year olds. Thus, precision and accuracy of CGM in the pediatric population with diabetes now approach those in adults with diabetes. In addition, device safety has been documented.

Notably, almost all patients studied had T1D (except for a single insulin-treated patient with type 2 diabetes in Study 1). Patients with T1D, in general, and pediatric patients, in particular, display substantially more glycemic variability than do adults. ${ }^{19,31}$ Despite the inherent glycemic challenges experienced by these young patients, the performance of the SW505 system is sufficient to provide opportunities to optimize glycemic control in the pediatric population, given its enhanced performance characteristics. Indeed, pediatric patients will benefit from opportunities to increase glucose time in range while reducing risk of severe hypoglycemia.

Previous studies of CGM in the pediatric population have yielded inconsistent results regarding the ability of CGM to improve glycemic control, measured as HbA1c, in the pediatric population. The most common observation to date regarding CGM efficacy among pediatric patients relates to the lack of durability of CGM use. Although many pediatric patients may initiate CGM, numerous studies have documented the lack of sustained use among these young patients. ${ }^{9-14}$ Discontinuation has been related to the perceived burden or hassle factors of CGM overwhelming any potential benefits. ${ }^{17,18,21,32-34}$ These burdens arise from the device's size and appearance, pain associated with insertion and 
wearing, frequent alerts and alarms, and inherent inaccuracies in earlier systems. The alerts and alarms, often referred to as "nuisance" alarms, become particularly annoying when the SMBG value, obtained to validate the CGM reading, does not match the CGM glucose value. Thus, there has been a need for improved accuracy of devices that can reduce this perceived nuisance factor. The G4P with SW505 offers greater accuracy and alert performance, thereby providing a real opportunity to minimize this nuisance factor.

In addition, the improved CGM performance in the hypoglycemic range, including values between 40 and $80 \mathrm{mg} / \mathrm{dL}$, may be able to provide reassurance that glycemic control can improve in the absence of an increased risk of severe hypoglycemia. Indeed, earlier pediatric studies have cited fear of hypoglycemia as a likely mediator of the previous failure of CGM to improve glycemic control in pediatric patients, especially in the younger patients, as parents focus on eliminating lower glucose levels in exchange for overall improvements in glycemia and target HbA1c attainment. ${ }^{13,14,35}$

A previous series of studies evaluated implementing CGM along with insulin pump therapy compared with multiple daily insulin injections (MDI) with SMBG in pediatric and adults patients with T1D. ${ }^{36-38}$ In these studies, known as STAR 3 (Sensor-Augmented Pump Therapy for A1C Reduction 3), using the Paradigm ${ }^{\circledR}$ REAL-time system from Medtronic (Northridge, CA), the pediatric patients, 7-18 years of age, who were randomized to sensor-augmented pump therapy compared with those randomized to MDI had significantly lower HbA1c levels, and a greater proportion of patients reaching age-specific glycemic targets had reduced glycemic variability, without increased hypoglycemia. ${ }^{37}$ Notably, the children, 7-12 years of age, used CGM more often than the adolescents, 13-18 years of age, in the 1-year randomized controlled trial, although both age groups benefited from the sensor-augmented pump therapy. During the 6-month extension phase of the study, the MDI group crossed over to sensoraugmented pump therapy and experienced significant improvements in HbA1c levels. ${ }^{38}$

The pediatric component of STAR 3 demonstrated opportunities for CGM implemented along with pump therapy to benefit the pediatric population. On the other hand, in the JDRF CGM study, which used multiple CGM devices, CGM use in the pediatric patients was not clearly associated with benefit in the younger patients, although adults derived significant benefit in that trial. ${ }^{5}$ Thus, in order for CGM to improve glycemic control without increased hypoglycemia in the pediatric population, CGM devices likely need to demonstrate improved performance or possibly be implemented with pump therapy. Additional opportunities may exist with the recent availability of wireless CGM data transmission to parents or other care providers, using systems such as the mySentry $^{\mathrm{TM}}$ from Medtronic ${ }^{39}$ or Dexcom Share ${ }^{\mathrm{TM}}{ }^{40}$

The studies described in the current report were short term and aimed at demonstrating safety and efficacy in order to achieve regulatory approval for use in the pediatric population. Longer-term studies are needed to assess whether the substantially improved performance of the SW505 algorithm results in greater uptake, sustained use, and improvements in glycemic control without an increase in severe hypoglycemia. Additionally, recent advancements in mobile communications with Bluetooth ${ }^{\circledR}$ (Bluetooth SIG, Kirkland, WA) connectivity that allows for transmission of CGM data directly to a smartphone, which eliminates the need to carry a separate receiver and then to transmit data directly to remote care providers, offer further opportunities to safely improve glycemic control in youth. These advances will likely promote increased uptake and durability of CGM use in pediatric patients; future studies are needed. Finally, use of the CGM data to make diabetes treatment decisions, including insulin dosing, will certainly reduce management burdens that are placed on patients and their families. Indeed, the Dexcom G4 Platinum with SW505 and the Dexcom G5 Mobile with SW505 have received the European CE mark for use in patients 2 years of age and older for nonadjunctive use, heralding in a potentially new era for diabetes management.

\section{Acknowledgments}

I would like to thank the participating sites, study staff, and the patients, acknowledging the site physicians and the biostatistician from Dexcom: Andrew Ahmann, MD, Portland, OR; Timothy Bailey, MD, Clinical Research, Advanced Metabolic Care and Research Institute, Escondido, CA; Bruce Buckingham, MD, Stanford University School of Medicine, Palo Alto, CA; Peter Chase, MD, Pediatrics, Barbara Davis Center for Diabetes, Denver, CO; Mark Daniels, MD, Children's Hospital of Orange County, Orange, CA; David Liljenquist, MD, Diabetes and Osteoporosis Center, Rocky Mountain, Idaho Falls, ID; Eva Tsalikian, MD, UIHC Department of Pediatrics, University of Iowa, Iowa City, IA; and Katherine Nakamura, PhD, Dexcom, San Diego, CA. The clinical studies were funded by Dexcom.

\section{Author Disclosure Statement}

L.L. reports consultancy for Johnson \& Johnson, Eli Lilly, Sanofi, NovoNordisk, Roche Diagnostics, Dexcom, AstraZeneca, and Boehringer Ingelheim.

\section{References}

1. Petitti DB, Klingensmith GJ, Andrews JS, Bell RA, Dabelea D, Imperatore G, Linder B, Loots B, Marcovina SM, Pihoker C, Rodriguez BL, Standiford DA, Waitzfelder B, Mayer-Davis EJ; SEARCH for Diabetes in Youth Study: Glycemic control in youth with diabetes: the SEARCH for Diabetes in Youth Study. J Pediatr 2009;155:668-672.

2. Beck RW, Tamborlane WV, Bergenstal RM, Miller KM, DuBose SN, Hall CA; T1D Exchange Clinic Network: The T1D Exchange clinic registry. J Clin Endocrinol Metab 2012;97:4383-4389.

3. Wood JR, Miller KM, Maahs DM, Beck RW, DiMeglio LA, Libman IM, Quinn M, Tamborlane WV, Woerner SE; T1D Exchange Clinic Network: Most youth with type 1 diabetes in the T1D Exchange Clinic Registry do not meet American Diabetes Association or International Society for Pediatric and Adolescent Diabetes clinical guidelines. Diabetes Care 2013;36:2035-2037.

4. Miller KM, Foster NC, Beck RW, Bergenstal RM, DuBose SN, DiMeglio LA, Maahs DM, Tamborlane WV; T1D Exchange Clinic Network: Current state of type 1 diabetes treatment in the U.S.: updated data from the T1D Exchange clinic registry. Diabetes Care 2015;38:971-978.

5. Juvenile Diabetes Research Foundation Continuous Glucose Monitoring Study Group: Continuous glucose monitoring and intensive treatment of type 1 diabetes. $\mathrm{N}$ Engl $\mathrm{J}$ Med 2008;359:1464-1476. 
6. Juvenile Diabetes Research Foundation Continuous Glucose Monitoring Study Group: The effect of continuous glucose monitoring in well-controlled type 1 diabetes. Diabetes Care 2009;32:1378-1383.

7. Juvenile Diabetes Research Foundation Continuous Glucose Monitoring Study Group: Effectiveness of continuous glucose monitoring in a clinical care environment: evidence from the Juvenile Diabetes Research Foundation continuous glucose monitoring (JDRF-CGM) trial. Diabetes Care 2010;33:17-22.

8. Battelino T, Conget I, Olsen B, Schütz-Fuhrmann I, Hommel E, Hoogma R, Schierloh U, Sulli N, Bolinder J; SWITCH Study Group: The use and efficacy of continuous glucose monitoring in type 1 diabetes treated with insulin pump therapy: a randomised controlled trial. Diabetologia 2012;55:3155-3162.

9. Diabetes Research in Children Network (DirecNet) Study Group: Continuous glucose monitoring in children with type 1 diabetes. J Pediatr 2007;151:388-393.

10. Diabetes Research in Children Network (DirecNet) Study Group: FreeStyle Navigator continuous glucose monitoring system use in children with type 1 diabetes using glarginebased multiple daily dose regimens: results of a pilot trial. Diabetes Care 2008;31:525-527.

11. Diabetes Research in Children Network (DirecNet) Study Group: Prolonged use of continuous glucose monitors in children with type 1 diabetes on continuous subcutaneous insulin infusion or intensive multiple-daily injection therapy. Pediatr Diabetes 2009;10:91-96.

12. Chase HP, Beck RW, Xing D, Tamborlane WV, Coffey J, Fox LA, Ives B, Keady J, Kollman C, Laffel L, Ruedy KJ: Continuous glucose monitoring in youth with type 1 diabetes: 12-month follow-up of the Juvenile Diabetes Research Foundation continuous glucose monitoring randomized trial. Diabetes Technol Ther 2010;12:507-515.

13. Mauras N, Beck R, Xing D, Ruedy K, Buckingham B, Tansey M, White NH, Weinzimer SA, Tamborlane W, Kollman C; Diabetes Research in Children Network (DirecNet) Study Group: A randomized clinical trial to assess the efficacy and safety of real-time continuous glucose monitoring in the management of type 1 diabetes in young children $(4$ to $<10$ year olds). Diabetes Care 2012;35:204-210.

14. Tsalikian E, Fox L, Weinzimer S, Buckingham B, White NH, Beck R, Kollman C, Xing D, Ruedy K; Diabetes Research in Children Network (DirecNet) Study Group: Feasibility of prolonged continuous glucose monitoring in toddlers with type 1. Pediatr Diabetes 2012;13:294-300.

15. Deiss D, Bolinder J, Riveline JP, Battelino T, Bosi E, Tubiana-Rufi N, Kerr D, Phillip M: Improved glycemic control in poorly controlled patients with type 1 diabetes using real-time continuous glucose monitoring. Diabetes Care 2006;29:2730-2732.

16. Juvenile Diabetes Research Foundation Continuous Glucose Monitoring Study Group: Factors predictive of use and of benefit from continuous glucose monitoring in type 1 diabetes. Diabetes Care 2009;32:1947-1953.

17. Tansey M, Laffel L, Cheng J, Beck R, Coffey J, Huang E, Kollman C, Lawrence J, Lee J, Ruedy K, Tamborlane W, Wysocki T, Xing D; JDRF CGM Study Group: Satisfaction with continuous glucose monitoring in adults and youths with Type 1 diabetes. Diabet Med 2011;28:1118-1122.

18. Ramchandani N, Arya S, Ten S, Bhandari S: Real-life utilization of real-time continuous glucose monitoring: the complete picture. J Diabetes Sci Technol 2011;5:860-870.
19. Juvenile Diabetes Research Foundation Continuous Glucose Monitoring Study Group, Wilson DM, Xing D, Beck RW, Block J, Bode B, Fox LA, Hirsch I, Kollman C, Laffel L, Ruedy KJ, Steffes M, Tamborlane WV: Hemoglobin A1c and mean glucose in patients with type 1 diabetes: analysis of data from the Juvenile Diabetes Research Foundation continuous glucose monitoring randomized trial. Diabetes Care 2011;34: 540-544.

20. Diabetes Control and Complications Trial Research Group: Effect of intensive diabetes treatment on the development and progression of long-term complications in adolescents with insulin-dependent diabetes mellitus: Diabetes Control and Complications Trial. J Pediatr 1994;125: 177-188.

21. Polonsky WH, Hessler D: Perceived accuracy in continuous glucose monitoring: understanding the impact on patients. J Diabetes Sci Technol 2015;9:339-341.

22. Damiano ER, McKeon K, El-Khatib FH, Zheng H, Nathan DM, Russell SJ: A comparative effectiveness analysis of three continuous glucose monitors: the Navigator, G4 Platinum, and Enlite. J Diabetes Sci Technol 2014;8:699-708.

23. Christiansen M, Bailey T, Watkins E, Liljenquist D, Price D, Nakamura K, Boock R, Peyser T: A new-generation continuous glucose monitoring system: improved accuracy and reliability compared with a previous-generation system. Diabetes Technol Ther 2013;15:881-888.

24. Johnson AW, Goodwin BF: The Draize test and modifications. Curr Probl Dermatol 1985;14:31-38.

25. Bland JM, Altman DG: Statistical methods for assessing agreement between two methods of clinical measurement. Lancet 1986;1:307-310.

26. Clarke WL, Cox D, Gonder-Frederick LA, Carter W, Pohl SL: Evaluating clinical accuracy of systems for self-monitoring of blood glucose. Diabetes Care 1987;10:622-628.

27. Parkes JL, Slatin SL, Pardo S, Ginsberg BH: A new consensus error grid to evaluate the clinical significance of inaccuracies in the measurement of blood glucose. Diabetes Care 2000;23:1143-1148.

28. Bailey TS, Chang A, Christiansen M: Clinical accuracy of a continuous glucose monitoring system with an advanced algorithm. J Diabetes Sci Technol 2015;9:209-214.

29. Russell SJ, El-Khatib FH, Sinha M, Magyar KL, McKeon K, Goergen LG, Balliro C, Hillard MA, Nathan DM, Damiano ER: Outpatient glycemic control with a bionic pancreas in type 1 diabetes. N Engl J Med 2014;371:313-325.

30. Kovatchev BP, Renard E, Cobelli C, Zisser HC, Keith-Hynes $\mathrm{P}$, Anderson SM, Brown SA, Chernavvsky DR, Breton MD, Mize LB, Farret A, Place J, Bruttomesso D, Del Favero S, Boscari F, Galasso S, Avogaro A, Magni L, Di Palma F, Toffanin C, Messori M, Dassau E, Doyle FJ 3rd: Safety of outpatient closed-loop control: first randomized crossover trials of a wearable artificial pancreas. Diabetes Care 2014;37: 1789-1796.

31. Xing D, Kollman C, Beck RW, Tamborlane WV, Laffel L, Buckingham BA, Wilson DM, Weinzimer S, Fiallo-Scharer $\mathrm{R}$, Ruedy KJ; Juvenile Diabetes Research Foundation Continuous Glucose Monitoring Study Group: Optimal sampling intervals to assess long-term glycemic control using continuous glucose monitoring. Diabetes Technol Ther 2011;13:351-358.

32. Diabetes Research in Children Network (DirecNet) Study Group: Youth and parent satisfaction with clinical use of the Glucowatch G2 Biographer in the management of pediatric type 1 diabetes. Diabetes Care 2005;28:1929-1935. 
33. Diabetes Research in Children Network (DirecNet) Study Group: Psychological aspects of continuous glucose monitoring in pediatric type 1 diabetes. Pediatr Diabetes 2006; 7:32-38.

34. Markowitz JT, Pratt K, Aggarwal J, Volkening LK, Laffel LM: Psychosocial correlates of continuous glucose monitoring use in youth and adults with type 1 diabetes and parents of youth. Diabetes Technol Ther 2012;14:523-526.

35. Tansey M, Weinzimer S, Beck R, Ruedy K, Cheng P, Tamborlane W, Kollman C, Mauras N, Fox L, Coffey J, White NH: Extended 6-month follow-up of a randomized clinical trial to assess the efficacy and safety of real-time continuous glucose monitoring in the management of type 1 diabetes in young children aged 4 to $<10$ years. Diabetes Care 2013;36:e63.

36. Bergenstal RM, Tamborlane WV, Ahmann A, Buse JB, Dailey G, Davis SN, Joyce C, Peoples T, Perkins BA, Welsh JB, Willi SM, Wood MA; STAR 3 Study Group: Effectiveness of sensor-augmented insulin-pump therapy in type 1 diabetes. N Engl J Med 2010;363:311-320.

37. Slover RH, Welsh JB, Criego A, Weinzimer SA, Willi SM, Wood MA, Tamborlane WV: Effectiveness of sensoraugmented pump therapy in children and adolescents with type 1 diabetes in the STAR 3 study. Pediatr Diabetes 2012;13:6-11.
38. Bergenstal RM, Tamborlane WV, Ahmann A, Buse JB, Dailey G, Davis SN, Joyce C, Perkins BA, Welsh JB, Willi SM, Wood MA; STAR 3 Study Group: Sensor-augmented pump therapy for A1C reduction (STAR 3) study: results from the 6-month continuation phase. Diabetes Care 2011;34:2403-2405.

39. Kaiserman K, Buckingham BA, Prakasam G, Gunville F, Slover RH, Wang Y, Nguyen X, Welsh JB: Acceptability and utility of the mySentry remote glucose monitoring system. J Diabetes Sci Technol 2013;7:356-361.

40. Dexcom. www.dexcom.com/dexcom-g4-platinum-share (accessed December 8, 2015).

Address correspondence to: Lori Laffel MD, MPH Pediatric, Adolescent and Young Adult Section Genetics and Epidemiology Section Joslin Diabetes Center One Joslin Place Boston, MA 02215

E-mail: Lori.Laffel@ Joslin.Harvard.Edu 